\title{
Síndrome de Regresión Caudal. Caso Clínico
}

\author{
MARÍA JOSÉ LUQUE H. ${ }^{1}$, ROCÍO FERNÁNDEZ B. ${ }^{1}$, MARÍA JESÚS TUCA D. ${ }^{1}$, \\ MATÍAS LUCO I. ${ }^{3}$, FLORENCIA DE BARBIERI M. ${ }^{2}$, JOSÉ LUIS TAPIA I. ${ }^{3}$ \\ 1. Médico, Escuela de Medicina, Facultad de Medicina, Pontificia Universidad Católica de Chile. \\ 2. Departamento de Radiología, Unidad de Radiología Pediátrica, Facultad de Medicina, Pontificia Universidad Católica \\ de Chile. \\ 3. Departamento de Pediatría, Unidad de Neonatología, Facultad de Medicina, Pontificia Universidad Católica de Chile, \\ Santiago, Chile.
}

\begin{abstract}
Caudal Regression Syndrome: Clinical Case and Update

Caudal regression is a rare congenital malformation which includes a wide spectrum of musculoskeletal abnormalities involving the lumbosacral spine, pelvis and lover limbs. It can be associated to visceral defects (gastrointestinal, genitourinary, cardiac or neurological) in various degrees. The etiology is not yet clear, but maternal diabetes, genetic predisposition, and vascular hypoperfusion are suspected. Objective: Describe a case of exceptional extension, including first year evolution. Clinical Case: A male newborn, term, was diagnosed before birth. Mother is a Type 2 diabetic. Physical exam and images confirm the diagnosis, show presence of 7 cervical vertebrae, 8 thoracic, agenesia of distal dorsal and lumbosacral spine. No spinal disraphia, medular conus at D2. Conclusion: The reported case shows that early diagnosis and multidisciplinary evaluation of the patient are essential elements to decrease complications and improve prognosis.
\end{abstract}

(Key words: Caudal regression syndrome, caudal dysplasia, sacral agenesis, diabetes, spinal dysraphia).

Rev Chil Pediatr 2010; 81 (2): 148-154

\section{RESUMEN}

Antecedentes: El síndrome de regresión caudal es una malformación congénita poco frecuente, caracterizada por un amplio espectro de anormalidades musculoesqueléticas que comprometen columna lumbosacra, pelvis y extremidades inferiores. Se puede asociar a diversos defectos viscerales (gastrointestinales, genitourinarios, cardíacos y neurológicos) presentes en distintos grados según la severidad del caso. Su etiología aún no se encuentra bien dilucidada, pero se sospecha que la diabetes materna, la predisposición genética y la hipoperfusión vascular serían algunos de los factores involucrados en su patogénesis. Objetivo: Dar a conocer un caso de regresión caudal de extensión excepcional y describir su evolución durante el primer año de vida. Caso

Trabajo recibido el 27 de noviembre de 2009, devuelto para corregir el 28 de diciembre de 2009, segunda versión el 26 de enero de 2010, aceptado para publicación el 18 de febrero de 2010.

Correspondencia a:

José Luis Tapia I.

E-mail: jlta@med.puc.cl 
clínico: Se presenta el caso de un recién nacido de término, sexo masculino, hijo de madre diabética tipo 2, con diagnóstico antenatal de síndrome de regresión caudal. El examen físico y las imágenes confirman el diagnóstico y muestran la presencia de siete cuerpos vertebrales cervicales y sólo ocho torácicos, con agenesia de columna dorsal distal y lumbosacra, sin disrrafia espinal y cono medular en nivel de D2. Conclusión: El caso reportado demuestra que tanto el diagnóstico precoz como la evaluación multidisciplinaria del paciente, son pilares esenciales para disminuir el riesgo de complicaciones asociadas y mejorar su pronóstico.

(Palabras clave: Síndrome de regresión caudal, secuencia de displasia caudal, agenesia sacra, displasia caudal).

Rev Chil Pediatr 2010; 81 (2): 148-154

\section{Introducción}

El término síndrome de regresión caudal (SRC) fue por primera vez utilizado por Bernard Duhamel en el año $1961^{1}$, el cual describe este síndrome como un espectro de defectos congénitos que involucran diversos grados de anormalidades musculoesqueléticas de columna lumbosacra, pelvis y extremidades inferiores, como también alteraciones de los sistemas neurológico, genitourinario, gastrointestinal y cardíaco. Dentro del espectro que define el SRC es posible encontrar una variedad de malformaciones que van desde ano imperforado, agenesia sacra, ausencia de vertebras lumbares y torácicas, hasta el extremo más severo que son las malformaciones de órganos mayores y la fusión de las extremidades inferiores, defecto conocido como sirinomelia ${ }^{2}$.

Si bien la causa de este síndrome aún no está claramente establecida, se cree éste que sería resultado de un defecto en la inducción de los elementos caudales del embrión previo a la séptima semana de gestación ${ }^{3,4}$, y que la diabetes mellitus materna jugaría un rol importante en su patogénesis ${ }^{5-7}$. En la literatura existen varias teorías sobre el origen del SRC, dentro de las cuales se describen alteraciones de la neurolización primaria o secundaria ${ }^{8-10}$, trastornos mesodérmicos del extremo caudal de la cuerda embrionaria temprana ${ }^{8,11}$ y la alteración generalizada de la migración mesodérmica durante el período de estría primitiva ${ }^{8,12}$. La proximidad y yuxtaposición entre el intestino posterior en desarrollo, el aparato genitourinario, la notocorda, y estructuras neurales, explica la asociación conjunta de malformaciones de los sistemas neurológico, vertebral, anorectal, renal y genital, comúnmente observadas en el $\mathrm{SRC}^{8,13}$. Por otro lado, se cree que la sirinomelia y sus defectos asociados serían atribuibles a un robo de flujo que condicionaría una isquemia severa hacia el hemicuerpo distal ${ }^{3,14-16}$. En estos pacientes se ha observado un vaso aberrante derivado de la arteria vitelina que redirige sangre desde la aorta abdominal hacia la placenta por medio del cordón umbilical. Este vaso actúa finalmente como una única arteria umbilical que roba el flujo que debería llegar al extremo caudal del feto condicionando isquemia e hipoperfusión. Por este motivo, es que a pesar que la sirinomelia es usualmente considerada como la forma más severa de SRC, algunos autores sugieren que ambas condiciones conformarían entidades separadas debido a su patogenia distinta. En este sentido, Twickler et al, en el año 1993 describió por primera vez las diferencias entre estas dos condiciones después de examinar 4 casos de sirinomelia y 3 casos de $\mathrm{SRC}^{15}$. Dentro de las características descritas que definen la sirinomelia y la distinguen de un SRC se encuentran: la presencia de una arteria umbilical única, agenesia caudal más severa (extremidad inferior única o dos fusionadas), alteraciones renales severas e incluso letales (agenesia renal o displasia severa), ausencia de malformaciones anales y oligohidroamnios ${ }^{15}$.

En la literatura se describen varios casos de SRC con agenesia sacra y lumbosacra, sin embargo, la agenesia dorsolumbosacra, en la cual hay compromiso de vértebras torácicas 
distales, lumbares y sacras, ha sido raramente reportada como parte de este síndrome, siendo la mayoría de estos casos sólo descripciones de anatomía patológica en fetos abortados ${ }^{2,17,18}$. En este reporte presentamos un caso excepcional de agenesia caudal con compromiso dorsal y lumbosacro, el cual fue dado de alta de nuestra Unidad de Neonatología y actualmente cuenta con más de un año de vida. No hemos encontrado en la literatura ningún caso tan severo de SRC en el cual se haya descrito su evolución durante el primer año de vida.

\section{Caso Clínico}

Madre de 34 años con antecedente de diabetes gestacional durante su primer embarazo, luego del cual queda con diagnóstico de diabetes mellitus tipo 2 en tratamiento con Metformina. Durante un período intergenésico de 5 años, evolucionó con oligomenorrea y no utilizó método anticonceptivo. Su segundo embarazo fue controlado desde las 6 semanas de gestación, donde debido a la presencia de glicemias de ayuno elevadas (mayores a 300 $\mathrm{mg} / \mathrm{dl}$ ) se reemplazó el tratamiento hipoglicemiante oral por insulina. Durante el segundo trimestre se requirió incrementar la dosis de insulina para lograr estabilizar sus glicemias. En ecografía realizada a las 18 semanas de embarazo se detectó agenesia de la columna torácica distal y lumbosacra, con extremidades inferiores cortas y oligoamnios moderado. Ecografías posteriores confirmaron la ausencia de elementos osificados en la columna lumbosacra, como también ausencia de movimientos de las extremidades inferiores del feto. La longitud de sus fémures era compatible con una gestación de 31 semanas a las 37 semanas de gestación.

Nuestro paciente de sexo masculino nació a las 38 semanas de edad gestacional por medio de cesárea electiva. Nació en buenas condiciones generales (Apgar 7-9), pesó 2990 g, midió $37 \mathrm{~cm}$ y su perímetro cefálico midió $37 \mathrm{~cm}$. En el período de recién nacido inmediato evolucionó con dificultad respiratoria y requerimiento de oxígeno de hasta un 30\% por aproximadamente 24 horas, lo cual se in- terpretó como taquipnea transitoria del recién nacido. Al examen físico destacó evidente hipoplasia del hemicuerpo inferior, con extremidades inferiores en flexo-abducción, pterigeon poplíteo bilateral y pies angostos. Conjuntamente se evidenció ausencia de columna lumbosacra a la palpación, hipoplasia glútea y ano permeable con atonía de esfínter. Los testículos no se encontraron a la palpación de escroto ni canal inguinal, su falo midió más de $20 \mathrm{~mm}$ y presentó micción espontánea (figura 1). El examen neurológico mostró paciente alerta sin movilidad espontánea ni respuesta a estímulos en hemicuerpo inferior, con tono muscular abolido y ausencia de reflejos tendíneos profundos en extremidades inferiores. Sin embargo, los reflejos arcaicos y osteotendíneos del hemicuerpo superior estaban conservados.

Se realizaron diversas imágenes para determinar la extensión y severidad de las anomalías musculoesqueléticas presentes. La radiografía de columna total AP (figura 2) y lateral mostró la presencia de sólo siete vértebras cervicales y ocho dorsales, sin desarrollo de la columna hacia distal ni del sacro. Las tres últimas vértebras dorsales y las costillas presentaban anomalías congénitas. Además había fusión de ambos huesos ilíacos e isquion, y falta de desarrollo del pubis. La maduración ósea se encontraba dentro de los límites normales para la edad gestacional. La resonancia

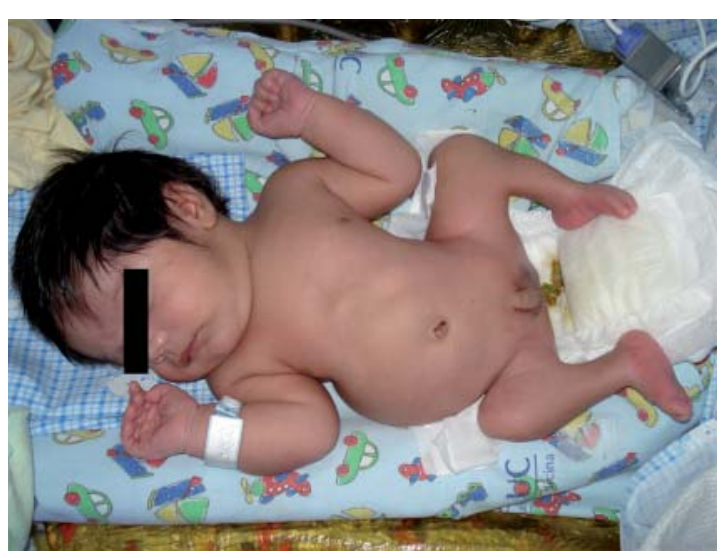

Figura 1. Fotografía del recién nacido con SDC. Se observa hipoplasia del hemicuerpo inferior, con extremidades inferiores en flexo-abducción, pterigeon poplíteo bilateral y pies angostos. 


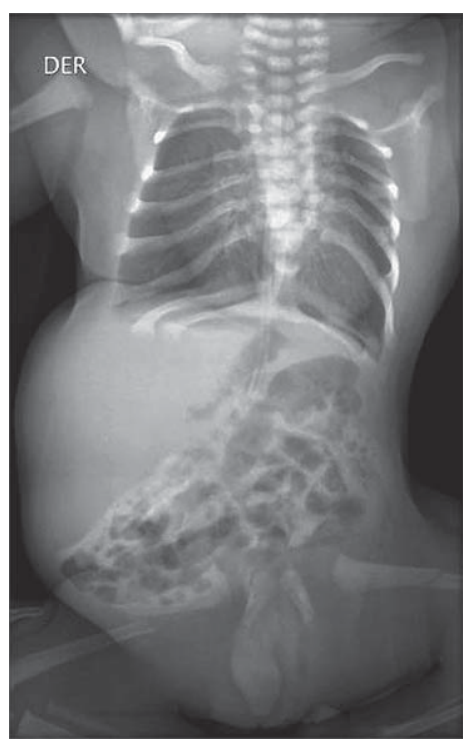

Figura 2. Radiografía de columna total AP-lateral: Son visibles siete vértebras cervicales y ocho dorsales. No hay desarrollo de la columna hacia distal ni del sacro. Las tres últimas vértebras dorsales y las costillas presentan anomalías congénitas. Hay fusión de ambos huesos ilíacos e isquion y falta de desarrollo del pubis. La maduración ósea está dentro de lo normal.

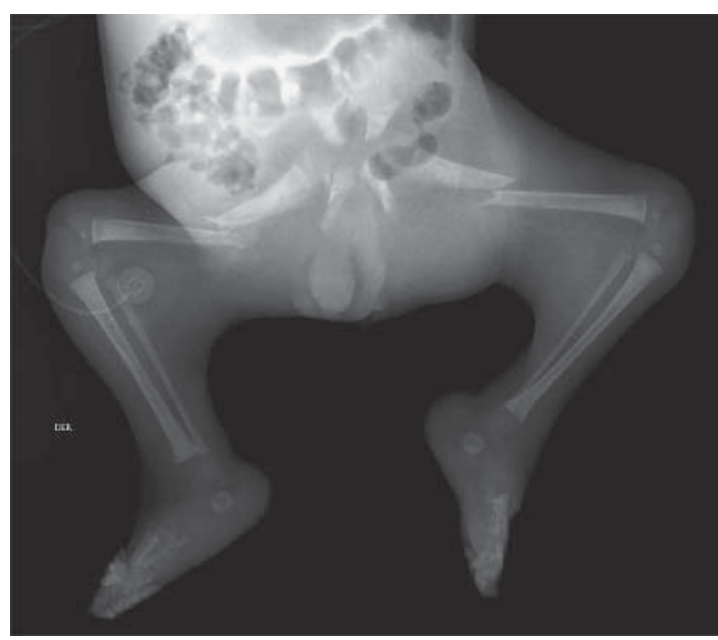

Figura 4. Radiografía de extremidades inferiores: Fractura de rasgo completo del tercio proximal de las diáfisis de ambos fémures con desplazamiento y cabalgamiento de los extremos.

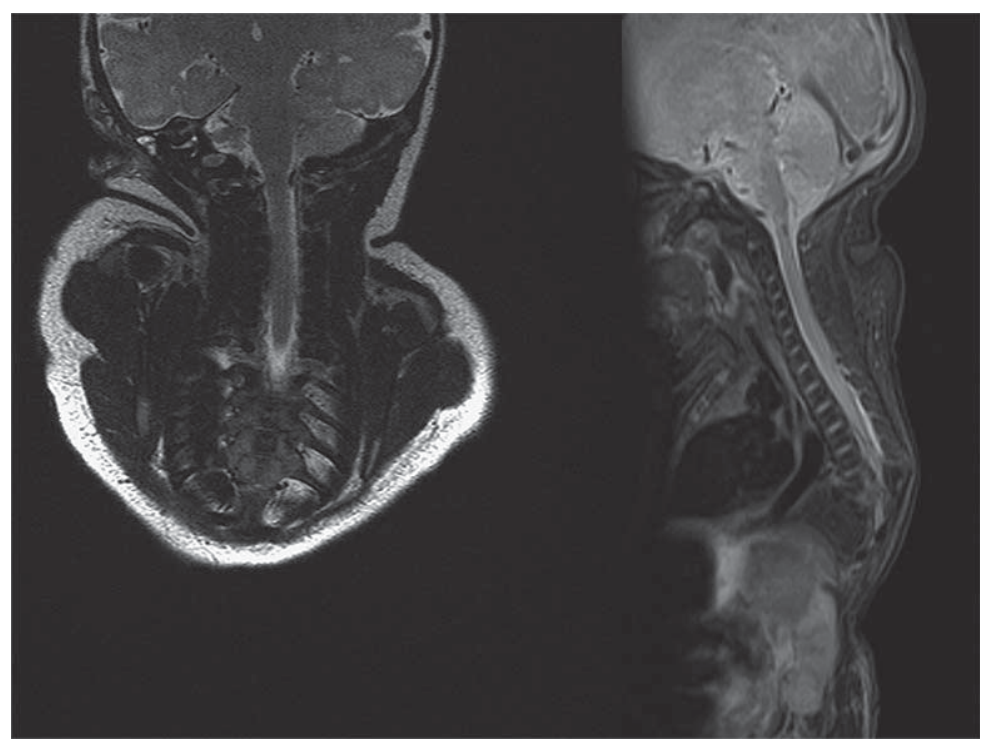

Figura 3. Resonancia magnética de columna total: Hay desarrollo de los cuerpos vertebrales hasta el nivel dorsal medio (D8). Hay agenesia de columna torácica distal y lumbosacra. Canal raquídeo de dimensiones normales. El extremo distal de la médula se encuentra a nivel de D3 y las raíces nerviosas se extienden hasta D8. No hay evidencias de disrrafias espinales ni de masas en el canal raquídeo. magnética de columna total (figura 3) mostró desarrollo de los cuerpos vertebrales hasta el nivel dorsal medio (D8), con agenesia de columna torácica distal y lumbosacra. El canal raquídeo tenía dimensiones normales y el extremo distal de la médula estaba a nivel de D3, con distribución normal de las raíces nerviosas hasta D8. No había evidencias de disrrafias espinales ni masas en el canal raquídeo. La radiografía de extremidades inferiores (figura 4) mostró fractura de rasgo completo del tercio proximal de las diáfisis de ambos fémures con desplazamiento y cabalgamiento de los extremos. Debido a esto fue evaluado por el equipo de ortopedia y traumatología infantil, quienes indicaron manejo conservador de las fracturas femorales, las cuales se encontraban en proceso de consolidación previo al alta.

Dentro del estudio se incluyó también una ecocardiografía doppler y una ecografía renalvesical con el fin de descartar otras malformaciones orgánicas asociadas. Los resultados de ambos exámenes no revelaron ninguna alteración anatómica significativa a nivel cardíaco ni 
nefro-urinario. De igual forma, la resonancia magnética de cerebro resultó normal.

Debido a la presencia de testículos no descendidos fue evaluado también por el equipo de endocrinología. Se solicitó cariograma el cual resultó normal (46 XY) y estudio hormonal (LH, FSH y Testosterona) que fue compatible con presencia de testículos, sin embargo, éstos no fueron hallados en la ecografía inguinal ni abdominal.

El paciente evolucionó favorablemente, sin alteraciones metabólicas. Fue dado de alta a los 16 días de vida, en buenas condiciones generales. Posteriormente, persistió en control multidisciplinario, incluyendo control ortopédico y urológico para seguimiento de infección urinaria y testículos no descendidos.

En su evaluación a los 13 meses de vida su desarrollo cognitivo y estado nutricional fueron normales y continuaba en terapia de neuro-rehabilitación motora en la Fundación Teletón. Desde el punto de vista genitourinario, sus testículos aún no habían descendido, presentaba vejiga neurogénica y había tenido sólo un episodio de infección urinaria tratada en forma ambulatoria con buena respuesta, quedando con profilaxis antibiótica y en control con nefrología para estudio y seguimiento. En la esfera gastrointestinal, evolucionó con constipación que se acompañó de impactación de fecalomas, por lo que se planteó probable intestino neurogénico, el cual se manejó con dieta alta en fibra y enemas evacuantes.

\section{Discusión}

El síndrome de regresión caudal (SRC), también conocido como secuencia de displasia caudal (SDC), es una anomalía congénita severa poco frecuente, caracterizada por la agenesia de extensión variable de los cuerpos vertebrales distales (sacrococcígeos o lumbosacrococcígeos), que se asocia frecuentemente a otras malformaciones musculoesqueléticas de la pelvis y extremidades inferiores ${ }^{8,19}$. De forma paralela, existen reportes de asociación conjunta con malformaciones viscerales, dentro de las cuales destacan alteraciones de los sistemas genitourinario, gastrointestinal, neu- rológico y cardíaco ${ }^{3}$. En la literatura se encuentran varios casos de SRC en los cuales la agenesia vertebral se extiende hasta nivel sacro y lumbosacro ${ }^{3,8}$, sin embargo, los casos de agenesia dorsolumbosacra reportados son muy escasos. Por este motivo, el caso presentado en este reporte resulta tan interesante de evaluar y comentar su evolución, ya que constituye un caso de SRC de extensión excepcional raramente descrita.

Tal como comentamos anteriormente, la presentación clínica de este síndrome puede incluir una larga lista de anormalidades. La agenesia sacra siempre va acompañada de caderas estrechas, hipoplasia glútea y aplanamiento del pliegue intergluteo. Los problemas ortopédicos pueden ir desde deformidades aisladas de los pies (pies estrechos) hasta deformidades complejas de las extremidades inferiores (hipoplasia femoral, contracturas en flexión, atrofia muscular). En casos aún más severos, como en los pacientes con sirinomelia, hay agenesia lumbosacra completa y fusión de extremidades inferiores. Las deformidades genitourinarias incluyen malformaciones renales (agenesia renal o hidronefrosis) y varias formas de duplicación de los ductos mullerianos. El déficit neurológico característicamente se expresa como paresia sensorio-motora por debajo el nivel medular normal, acompañado a veces también de vejiga neurogénica ${ }^{20}$. En el caso presentado tempranamente se detectaron varias de estas anormalidades evidentes al examen físico, todas concordantes con SRC.

El SRC es una malformación muy poco común en la población general, con una frecuencia estimada de 1: $7500 \mathrm{RN}$, sin diferencias por $\operatorname{sexo}^{20}$. La gran mayoría de los casos son esporádicos, sin embargo, existen reportes que sugieren la existencia de una contribución genética, al menos parcial, en el desarrollo de este síndrome ${ }^{3,8,14,21}$. Existen casos de SRC familiar, pero ningún patrón de herencia Mendeliana ha sido establecido ${ }^{22,23}$. Estudios recientes entregan evidencia sobre la participación de un gen "homeobox" (HLXB9) en el desarrollo de la agenesia sacra de herencia dominante, pero su posible relación con el origen del SRC no ha sido establecida ${ }^{22,23}$.

Numerosos factores ambientales han sido 
reportados como potenciales factores teratogénicos, dentro de ellos están la: hiperglicemia, hipoxia, anormalidades en cetonas o aminoácidos, glicosilación de proteínas, desbalances hormonales y otros (litio, déficit vitamina A, ácido retinoico, hipertemia, etc $)^{8,23,24}$. A pesar que aún no se ha encontrado una causa específica para el SRC, el teratógeno más comúnmente reconocido es la hiperglicemia, la cual creemos habría jugado un rol fundamental en este caso. Existen varios estudios que analizan esta asociación entre diabetes y SRC. Dentro de sus conclusiones destaca que los hijos de madres con diabetes pregestacional tendrían un riesgo al menos 200 veces mayor de padecer este síndrome ${ }^{5}$ y que el 16 a $22 \%$ de los niños con SRC son hijos de madres diabéticas ${ }^{3,8}$, todo lo cual convertiría al SRC en la malformación más característica de la embriopatía diabética ${ }^{2,3,8,24-26}$.

En el caso expuesto, está el antecedente de la madre con diabetes pre-gestacional tipo 2, con mal control metabólico, que requirió el uso de insulina durante el embarazo en dosis crecientes. La asociación de DM materna con anomalías congénitas es bien conocida. Los niños hijos de madres insulinodependientes tienen un riesgo aumentado de malformaciones congénitas, especialmente mayores, multiorgánicas. Se comprometen con mayor frecuencia los sistemas cardiovascular, SNC, genitourinario y esquelético. Este peor resultado neonatal se ha asociado históricamente a madres portadoras de diabetes pre-gestacional tipo 1, pero estudios recientes le dan un riesgo similar a las diabéticas pre-gestacionales tipo $2^{6,7,27,28}$. La placenta es una buena barrera contra la insulina materna, y el feto no produce insulina hasta al menos la octava semana de gestación. Por este motivo el feto se encuentra mucho más vulnerable a la hiperglicemia durante este período. De esta forma, la mantención de un buen control metabólico durante el período preconcepcional y durante todo el embarazo, especialmente durante el primer trimestre, es de esencial importancia para prevenir malformaciones congénitas ${ }^{8}$.

La importancia de éste síndrome radica en las complicaciones neurológicas, urológicas y ortopédicas que pueden tener a largo plazo, ya que el pronóstico de los pacientes con SRC va a depender fundamentalmente de la severidad de la lesión y de la presencia de alguna de estas anormalidades asociadas. En el caso expuesto, las principales complicaciones asociadas involucran el aparato genitourinario y gastrointestinal, manifestándose por vejiga neurogénica y constipación crónica secundaria a intestino neurogénico, sin otras malformaciones orgánicas. Gracias a un seguimiento estricto y multidisciplinario nuestro paciente se mantuvo relativamente estable durante su primer año de vida, presentando sólo un episodio de infección urinaria durante éste período, con un desarrollo psicomotor y nutricional normales para la edad. De este modo, es de vital importancia la detección temprana y una amplia evaluación de los niños con SRC para disminuir el riesgo de incontinencia, ITU recurrente, daño renal y vejiga neurogénica ${ }^{29,30}$. Generalmente los niños que sobreviven tienen una función cerebral normal y requieren de una extensa asistencia urológica y ortopédica ${ }^{31}$, tal como se llevó a cabo en el caso presentado.

\section{Referencias}

1.- Duhamel B: From the mermaid to anal imperforation: The syndrome of caudal regression. Arch Dis Child 1961; 36: 152-5.

2.- Aslan H, Yanik H, Celikaslan N, Yildirim G, Ceylan Y: Prenatal diagnosis of Caudal Regression Syndrome: a case report. BMC Pregnancy Childbirth 2001; 1 (1): 8.

3.- Stroustrup Smith A, Grable I, Levine D: Case 66: caudal regression syndrome in the fetus of a diabetic mother. Radiology 2004; 230: 229-33.

4.- Sadler TW: Langman's Medical Embryology. 8th ed. Philadelphia, Pa: Lippincott Williams \& Wilkins, 2000; 61-110.

5.- Yang J, Cummings EA, O'Connell C, et al: Fetal and neonatal outcomes of diabetic pregnancies. Obstet Gynecol 2006; 108: 644-50.

6.- Nazer J, García Huidobro M, Cifuentes L: Malformaciones congénitas en hijos de madres con diabetes gestacional. Rev Med Chile 2005; 133: 547-54.

7.- Macintosh M, Fleming KM, Bailey JA, et al: Perinatal mortality and congenital anomalies in babies of women with type 1 or type 2 diabetes in England, Wales, and Northern Ireland: population based study. BMJ 2006; 333 (7560): 177-81. 
8.- Zaw W, Stone DG: Caudal Regression Syndrome in twin pregnancy with type II diabetes. J Perinatol 2002; 22 (2): 171-4.

9.- Nievelstein RA, ValkJ, Smit LM, et al: MR of the Caudal Regression Syndrome: Embryologic implications. AJNR, Am J Neuroradiol 1994; 15: 1021-9.

10.- Pang D: Sacral agenesis and caudal spinal cord malformations. Neurosurgery 1993; 32: 755-78.

11.- Tortori-Donati P, Fondelli MP, Rossi A, et al: Segmental spinal dysgenesis: Neuroradiologic findings with clinical and embryologic correlation. AJNR, Am J Neuroradiol 1999; 20: 445-56.

12.- Depraetere M, Dehauwere R, Marien P, et al: Severe axial mesodermal dysplasia spectrum in an infant of a diabetic mother. Genet Counsel 1995; 6: 303-7.

13.- Naidich T, Zimmerman R, McLone DG, et al: Congenital anomalies of the spine and spinal cord: embryology and malformations. In: Atlas S, editor. Magnetic Resonance Imaging of the Brain and Spine. Philadelphia: Lippincott-Raven; 1996: 1265-337.

14.- Callen $P W$ : Ultrasonography in obstetrics and gynecology. 4th ed. Philadelphia, Pa: Saunders, 2000; 364-7.

15.- Twickler D, Budorick N, Pretorius D, et al: Caudal regression versus sirenomelia: Sonographic clues. J Ultrasound Med 1993; 12: 323-30.

16.- Benacerraf BR: Ultrasound of fetal syndromes. Philadelphia, Pa: Churchill Livingstone 1998; 250-4.

17.- Mihmanli I, Kuruoglu S, Kantarci F, Kanberoglu K: Dorsolumbosacral agenesis. Pediatr Radiol 2001; 31: 286-8.

18.- Nagy GR, Csapo Z, Barakonyi E, et al: Prenatal diagnosis and fetopathological investigation of dorsolumbosacral agenesis. Pathol Res Pract 2009; 205 (7): 490-3.

19.- Kalter H: Case report of malformations associated with maternal diabetes: history and critique. Clin Genet
1993; 43: 174-9.

20.- Unsinn KM, Geley T, Freund MC, Gassner I: US of the Spinal Cord in Newborns: Spectrum of Normal Findings, Variants, Congenital Anomalies, and Acquired Diseases. RadioGraphics 2000; 20: 923-38.

21.- Subtil D, Cosson M, Houfflin V, Vaast P, Valat A, Puech $F$ : Early detection of caudal regression syndrome: specific interest and findings in three cases. Eur J Obstet Gynecol Reprod Biol 1998; 80: 109-12.

22.- Das B, Rajegowda B, Bainbridge R, Giampietro P: Caudal Regression Syndrome Versus Sirenomelia: A Case Report. J Perinatol 2002; 22: 168-70.

23.- Ross AJ, Ruiz-Pérez V, Wang Y, et al: A homeobox gene, HLXB9, is the major locus for dominantly inherited sacral agenesis. Nat Genet 1998; 20: 358-61.

24.- Mills JL: Malformations in infants of diabetic mothers. Teratology 1984; 25: 385-94.

25.- Twining P, McHugo J, Pilling D: Textbook of fetal abnormalities. Philadelphia, Pa: Saunders 2000; 158-60.

26.- Gabbe SG, Niebyl JR, Simpson JL: Obstetrics: normal and problem pregnancies. 4th ed. New York, NY: Churchill Livingstone 2002; 1090-1.

27.- Sheffield JS, Butler-Koster EL, Casey BM, et al: Maternal diabetes mellitus and infant malformations. Obstet Gynecol 2002; 100: 925-30.

28.- Nold JL, Georgieff MK: Infants of diabetic mothers. Pediatr Clin North Am 2004; 51: 619-37.

29.- Samartzis D, Shen FH: Ann Acad Med Singapore 2008; 37 (5): 446.

30.- Wilmshurst JM, Kelly R, Borzyskowski M: Presentation and outcome of sacral agenesis: 20 years' experience. Dev Med Child Neurol 1999; 41: 806-12.

31.- Adra A, Cordero D, Mejides A, et al: Caudal regression syndrome: Etiopathogenesis, prenatal diagnosis, and perinatal management. Obstet Gynecol Sur 1994; 49: 508-16. 